Artigo Original

\title{
Efeito da altura do selim na cinemática da lombar de ciclistas
}

\author{
Julio Francisco Kleinpaul ${ }^{1}$ \\ Luana Mann 1 \\ Diogo Cunha dos Reis ${ }^{1}$ \\ Felipe Pivetta Carpes ${ }^{2}$ \\ Antônio Renato Pereira Moro ${ }^{1}$ \\ ${ }^{1}$ Laboratório de Biomecânica, Universidade Federal de Santa Catarina, \\ Florianópolis, SC, Brasil \\ ${ }^{2}$ Grupo de Pesquisa em Neuromecânica Aplicada, Universidade Federal do Pampa, \\ Uruguaiana, RS, Brasil
}

\begin{abstract}
Resumo: $O$ objetivo deste estudo foi analisar a influência da altura do selim sobre a cinemática da coluna lombar durante o ciclismo. Foram avaliados oito ciclistas recreacionais, na situação usual (auto ajustada) e na situação de selim ajustado, com carga de $150 \mathrm{~W}$, utilizando-se avaliação cinemática bidimensional em um ciclosimulador. Foram mensurados os ângulos de flexão, inclinação lateral e rotação da coluna lombar. A flexão da lombar foi maior na situação de selim ajustado, enquanto que a inclinação e a rotação da lombar foram maiores na situação de ajuste usual. Quanto ao comportamento ao longo do ciclo de pedalada, ocorreram dois ciclos de flexão-extensão durante um ciclo completo do pé-de-vela, e apenas um ciclo de inclinação lateral ou de rotação. As maiores diferenças acorreram no início, meio e fim do ciclo. $O$ ajuste equivocado do selim não acarreta alterações na cinemática da coluna lombar.
\end{abstract}

Palavras-chave: Ciclismo. Cinemetria. Coluna. Ergonomia.

\section{Effect of saddle height on the kinematics of lumbar spine of cyclists}

\begin{abstract}
The aim of this study was to analyze the influence of saddle height on the kinematics of the lumbar spine during cycling. Eight recreational cyclists were assessed in an usual situation (self-selected) and the situation of saddle properly adjusted while pedaling with a load set at $150 \mathrm{~W}$. Using two-dimensional kinematic the angles of flexion, lateral inclination and rotation of the lumbar spine were computed. The flexion was greater in the adjusted position whereas the highest average tilt and rotation angles occurred in the usual position. There were two cycles of flexion-extension observed during a complete pedaling cycle, and only one cycle of lateral inclination and rotation. The major differences were observed at early, middle and end of the pedaling cycle. The mismanagement of the saddle did not involve significant changes in the kinematics of the lumbar spine for the conditions tested.
\end{abstract}

Keywords: Cycling. Kinematic. Spine. Ergonomics.

\section{Introdução}

A prática do ciclismo não abrange somente ciclistas e triatletas profissionais, mas também a população em geral. Para estes, a bicicleta também é considerada um excelente meio de transporte e lazer (ROWE; HULL; WANG, 1998). $\mathrm{Na}$ prática do ciclismo, como forma de atividade física regular, é necessário levar em consideração a correta postura do ciclista, principalmente no que tange $o$ ajuste do selim. Isto é necessário para evitar desconfortos e dor, o que pode causar problemas na coluna, sendo este um dos fatores para o abandono deste esporte (MARTINS et al., 2007).

Os aspectos relacionados à ergonomia no ciclismo envolvem, principalmente, 0 ajuste correto da interação ciclista-bicicleta na busca tanto por conforto como por desempenho. Além dos prejuízos na técnica e na eficiência, ciclistas são regularmente confrontados com lesões e dores crônicas causadas por desvios na postura adequada na bicicleta, tais como o aumento da flexão de quadril e de joelho (BURKE, 1996), o que tem sido efetivamente minimizado quando 0 posicionamento é correto (BURKE, 1996; VAN HERP et al., 2000).

As análises cinemáticas da coluna têm sido conduzidas a fim de identificar fatores de risco para a ocorrência de lombalgia, que pode ser recorrente entre ciclistas. No ciclismo, a busca por um método padrão para análise cinemática da coluna lombar limita-se aos poucos estudos e 
metodologias desenvolvidas para tal (BRESSEL; LARSON, 2003; SAUER et al., 2007; USABIAGA et al., 1997). Acredita-se que a escassez de estudos deva-se a dificuldades metodológicas para quantificar a geometria vertebral em movimento e de forma precisa e acurada. Métodos tridimensionais que poderiam resultar em dados interessantes para a prevenção de lesões esbarram em dificuldades metodológicas, como fixação de muitos marcadores de referência, afetando significativamente o conforto do atleta e, consequentemente, a fidedignidade dos dados. Todavia, a mudança na posição de ajustes móveis da bicicleta, como é o caso do selim, tem se mostrado suficiente para alterar parâmetros biomecânicos da atividade, tais como alterações na atividade mioelétrica (DOREL; COUTURIER; HUG, 2007) e cinemática do membro inferior (GREGOR; RUGG, 1986). Nesse sentido, as medidas cinemáticas da coluna lombar também contribuem para análises do posicionamento corporal para o ciclismo recreacional e competitivo. Uma vez que diversas metodologias têm sido sugeridas, mas não aplicadas regularmente, o objetivo deste estudo foi analisar os efeitos de diferentes ajustes do selim sobre o comportamento angular da coluna lombar utilizando ferramentas de cinemetria bidimensional, que reduzem 0 volume de preparação do sujeito e podem ser aplicáveis para o contexto clínico e do treinamento.

Diante da importância da flexibilidade lombar como fator determinante do desempenho de um ato motor vital e da escassez metodológica para uma investigação que forneça dados quantitativos sobre a movimentação dos segmentos da coluna lombar durante o ciclismo, a hipótese formulada para este estudo foi de que a alteração da altura do selim influencia significativamente os movimentos nos planos sagital, frontal e transverso nos segmentos ósseos na região da coluna vertebral lombar, diminuindo as amplitudes angulares.

\section{Métodos}

\section{Participantes}

Foram avaliados oito ciclistas recreacionais do sexo masculino residentes na cidade de Florianópolis. A seleção dos sujeitos foi do tipo não probabilístico intencional e o estudo foi aprovado em comitê local de ética em pesquisa (registro número 208/2009). A Tabela 1 apresenta as características dos sujeitos avaliados.
Tabela 1. Características dos sujeitos avaliados: idade (anos), massa corporal $(\mathrm{kg})$ e estatura $(\mathrm{m})$.

\begin{tabular}{ccc}
\hline Idade (anos) & Massa $(\mathrm{kg})$ & Estatura $(\mathrm{m})$ \\
\hline $27,63 \pm 6,1$ & $73,81 \pm 6,8$ & $1,77 \pm 0,05$ \\
\hline
\end{tabular}

\section{Instrumentos}

Para aquisição dos dados foi utilizada uma bicicleta MTB Aerotech aro 27 e um ciclosimulador (CompuTrainer Pro Lab 3D, Racermate Inc., Seattle, EUA), sobre o qual a bicicleta foi montada. O ciclosimulador gerou a sobrecarga mecânica para o teste (potência fixa de $150 \mathrm{~W}$ ), que é característica do ciclismo recreacional (BURKE; PRUITT, 2003) e também permitiu monitorar a cadência de pedalada, que foi mantida constante (70 rpm). Uma fita métrica foi utilizada para medir dimensões da bicicleta e parâmetros antropométricos necessários aos procedimentos de avaliação. Um goniômetro (Carci Ind. Com. Equip. Cirurg. Ortop., São Paulo, Brasil) com resolução de $5^{\circ}$ foi utilizado para medir e ajustar os ângulos articulares do voluntário durante a posição estática na bicicleta. A avaliação cinemática bidimensional foi feita utilizando um software de análise postural gratuito e de domínio público (SAPO versão 0.68). As imagens foram processadas com o objetivo de determinar os ângulos a cada decil (10\%) do ciclo de revolução do pé-de-vela (PDV). Para extrapolar os dados e garantir que ocorresse 0 mesmo número de eventos em cada ciclo do PDV os dados foram normalizados pelo tempo do ciclo de menor duração. As imagens foram adquiridas com duas câmeras digitais (SONY HVR-V1U, Califórnia, EUA) sincronizadas, e que operaram com taxa de amostragem de $30 \mathrm{~Hz}$. As câmeras foram posicionadas paralelas ao plano frontal e sagital do movimento, permitindo a avaliação das imagens no plano frontal e no plano sagital, separadamente em cada uma das câmeras. As câmeras foram posicionadas paralelas ao plano de movimento de interesse e perpendiculares ao movimento. Assim, ângulos de flexão e extensão foram mensurados com imagens de uma câmera posicionada lateralmente ao sujeito, enquanto a câmera posicionada posteriormente ao sujeito serviu para cálculos no plano frontal.

A coluna lombar foi analisada em segmentos a partir do posicionamento de marcadores adesivos reflexivos em forma de hastes $(85 \mathrm{~mm}$ de comprimento e $5 \mathrm{~mm}$ de diâmetro), para permitir também a análise dos ângulos de rotação, posicionados sobre a pele seguindo 0 alinhamento dos processos espinhosos das vértebras, avaliados por palpação. Os pontos de 
referência anatômica demarcados foram: processos espinhosos da primeira a quinta vértebras lombares e primeira sacral, espinha ilíaca póstero superior direita (EIPS) e espinha ilíaca ântero superior direita (EIAS), que serviram para o cálculo dos ângulos avaliados. Os marcadores das espinhas ilíacas ântero e póstero superiores serviram para controlar o ângulo de inclinação da pelve (Figura 1). Foram analisadas posições dinâmicas (ciclista pedalando) assumindo diferentes posições do selim (usual e ajustado), testadas randomicamente.

\section{Procedimentos para a coleta dos dados}

A avaliação cinemática foi realizada em duas situações: posição usual do selim, correspondente àquela que 0 sujeito escolhia voluntariamente, e a posição do selim ajustada de acordo com parâmetros biomecânicos. Os procedimentos utilizados para as duas situações de coleta foram os mesmos, o que os diferenciou foi a altura do selim. Na situação que utilizou a posição usual do selim, o mesmo estava do mesmo modo que os ciclistas utilizam rotineiramente (altura do selim $69,75 \pm 2,51 \mathrm{~cm}$ ) e, na situação selim ajustado (altura do selim $75,56 \pm 3,57 \mathrm{~cm}$ ). A altura para o selim ajustado foi definida de acordo com o protocolo de Burke e Pruitt (2003).

Todos os sujeitos pedalaram em uma bicicleta durante um minuto após a estabilização da cadência de pedalada. Houve um período mínimo de acomodação de $30 \mathrm{~s}$ após ser atingida a cadência de $70 \mathrm{rpm}$, em cada posição, antes da coleta dos dados cinemáticos.

Cinco ciclos em cada situação de teste foram selecionados para análise cinemática 2D, realizada através do Software de Análise Postural (SAPO) versão 0.68.

\section{Obtenção das coordenadas dos pontos da coluna lombar}

O método para a descrição dos movimentos da coluna lombar consistiu nas seguintes etapas: (a) construção de um modelo de representação da coluna lombar como um sistema de corpos rígidos articulados, sendo utilizado um conjunto de 13 marcadores posicionados sobre os acidentes ósseos da região lombar; (b) obtenção das coordenadas espaciais dos marcadores por meio da digitalização das imagens; (c) determinação dos ângulos propostos para a avaliação da mobilidade nesta região da coluna vertebral. a)

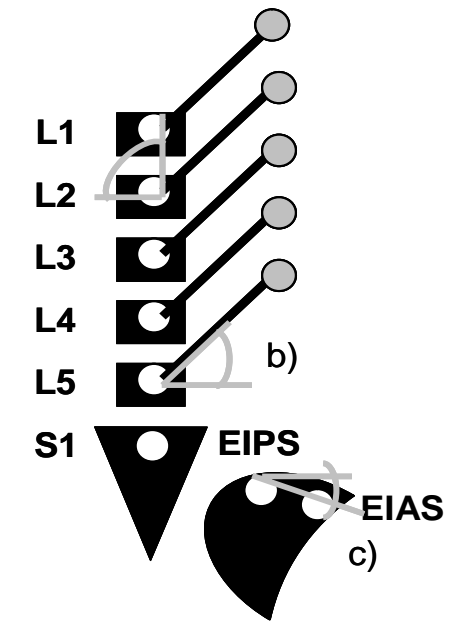

Figura 1. Cálculo das variáveis angulares (L1-L2, L2-L3, L3-L4, L4-L5, L5-S1). Esquema dos ângulos a serem calculados nos três planos a) flexão-extensão no plano sagital e inclinação lateral no plano frontal; b) rotação no plano frontal; c) inclinação pélvica no plano sagital.

As imagens adquiridas foram digitalizadas considerando os pontos anatômicos de referência para a análise do movimento angular da coluna lombar durante a pedalada. Após a digitalização das imagens e determinação dos ângulos, os dados foram filtrados em ambiente Matlab versão 7.0.1 (MathWorksInc, USA) através de um filtro passa-baixa do tipo Butterworth de segunda ordem e frequência de corte de $6 \mathrm{~Hz}$ (BRESSEL; LARSON, 2003). Os dados foram analisados em função do ângulo do PDV utilizando o programa Origin 6.0 Professional (Microcal., Inc., EUA).

\section{Análise estatística}

Todos os procedimentos estatísticos foram realizados no pacote estatístico PASW 15.0 (SPSS inc) (StatisticalPackage for the Social Sciences) e SISVAR 5.0. Utilizou-se estatística descritiva para medidas de tendência central (média) e de dispersão (desvio-padrão) na apresentação dos dados angulares, antropométricos (massa e estatura) e da altura do selim nas duas situações de ajuste. A fim de verificar se os dados possuíam distribuição normal, aplicou-se o teste de Shapiro-Wilk, o qual comprovou a normalidade dos mesmos. A esfericidade dos dados foi verificada pelo teste de Mauckly. A comparação da altura do selim nas duas situações e o comportamento angular lombar durante o ciclo de pedalada nas duas posições foi feita por Análise de Variância em um modelo linear misto (2 ajustes, 11 decis) com correções de Bonferroni para comparações múltiplas. O Teste $t$ de Student pareado foi aplicado para comparar a altura média do selim nas situações usual e ajustado. Os resultados de 
todas as análises foram considerados significativos quando $p<0,05$.

\section{Resultados}

A Tabela 2 apresenta as alturas médias do selim nas situações usual e ajustado.

Tabela 2. Altura média do selim nas situações usual e ajustado $(\mathrm{cm})$.

\begin{tabular}{lll}
\hline \multicolumn{3}{c}{ Altura do selim } \\
\hline Desajustado $(\mathrm{cm})$ & Ajustado $(\mathrm{cm})$ & $\mathrm{p}^{*}$ \\
\hline $69,75 \pm 2,51$ & $75,56 \pm 3,57$ & 0,001 \\
\hline
\end{tabular}

Os dados mostram que, para flexão da pelve, houve efeito do ajuste $\left[F_{(1,7)}=7,198 ; p=0,031\right]$; e não houve interações significativas entre ajuste $e$ decil $\left[F_{(10,70)}=1,843 ; p=0,069\right]$.

A Figura 2 apresenta os ângulos encontrados para a pelve, no plano sagital, nas situações de selim usual e ajustado. Com exceção do segundo, terceiro e quarto decis do ciclo de pedalada (10, 20 e $30 \%$ do ciclo), todos os demais momentos (40-100\% do ciclo de pedalada) apresentaram maiores valores de flexão na posição ajustada do que na posição usual $(\mathrm{p} 1=0,008 ; \mathrm{p} 2=0,145$; p3 $=0,242 ; \quad p 4=0,128 ; \quad p 5=0,028 ; \quad p 6=0,014$; p7 $=0,018 ; \quad p 8=0,028 ; \quad p 9=0,037 ; \quad p 10=0,028$ p11=0,005). Na Figura 2 pode-se observar que, principalmente na posição ajustada, ocorreram dois ciclos de flexão-extensão ao longo de um ciclo do PDV (comportamento em senóide).

\section{Pelve}

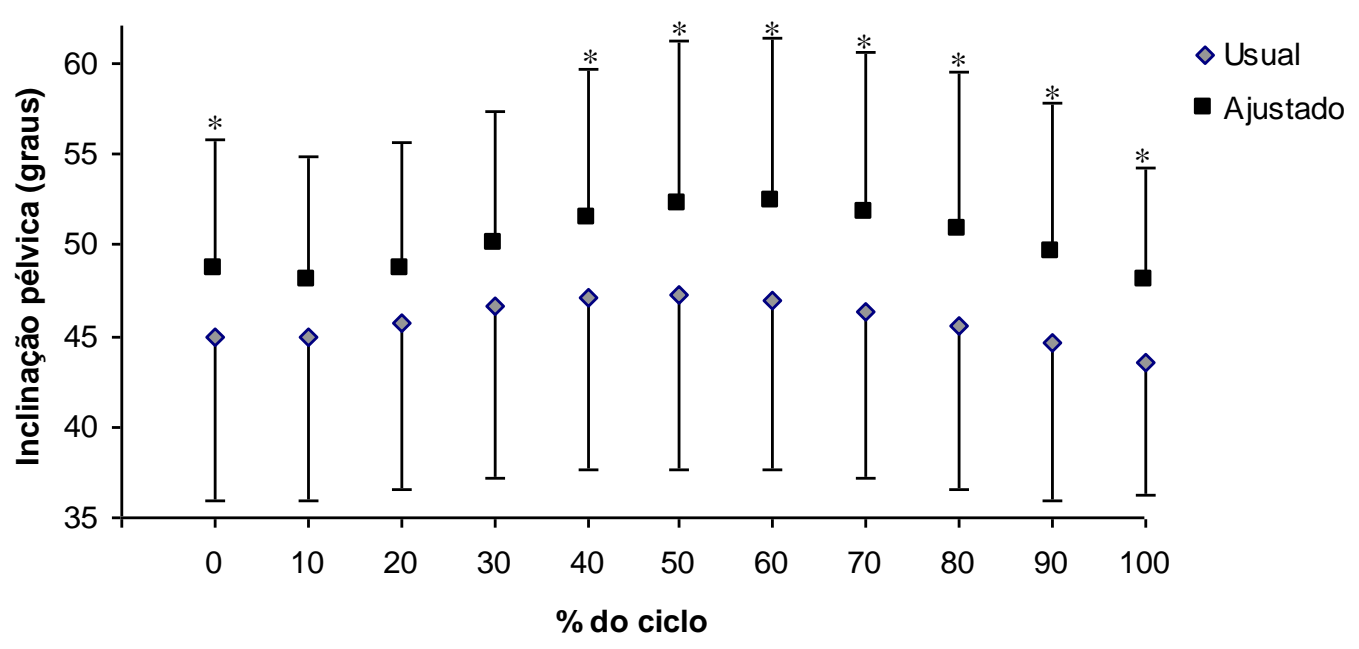

Figura 2. Comportamento angular da pelve no plano sagital em função do ciclo do pé-de-vela nas duas posições adotadas. ${ }^{*} p<0,05$ representa valor estatisticamente significativo (Bonferroni) entre as situações de selim ajustado e usual.

A Figura 3 indica os decis do ciclo do PDV em que foram encontradas diferenças significativas entre a posição usual do selim e posição de selim ajustado para a coluna lombar no plano sagital. Não foram encontradas diferenças estatisticamente significativas, principalmente a 70,80 e $90 \%$ do ciclo de PDV.

Os dados mostram que, para flexão/extensão do segmento $L 1 L 2$, houve efeito do ajuste $\left[F_{(1,7)}=\right.$ 11,278; $p=0,012$ ]; e interações significativas entre ajuste e decil $\left[F_{(10,70)}=2,248 ; p=0,024\right]$. Para flexão/extensão do segmento L2L3, houve efeito do ajuste $\left[F_{(1,7)}=12,849 ; p=0,009\right]$; e não houve interações significativas entre ajuste e decil $\left[F_{(10,70)}=0,527 ; p=0,845\right]$. Para flexão/extensão do segmento $L 3 L 4$, houve efeito do ajuste $\left[F_{(1,7)}=\right.$ 8,453; $p=0,023]$; e não houve interações significativas entre ajuste e decil $\left[F_{(10,70)}=1,017\right.$; $p=0,439]$. Para flexão/extensão do segmento $L 4 L 5$, houve efeito do ajuste $\left[F_{(1,7)}=11,866\right.$; $p=0,011]$; e não houve interações significativas entre ajuste e decil $\left[F_{(10,70)}=0,878 ; p=0,557\right]$. 
Segmento L1-L2
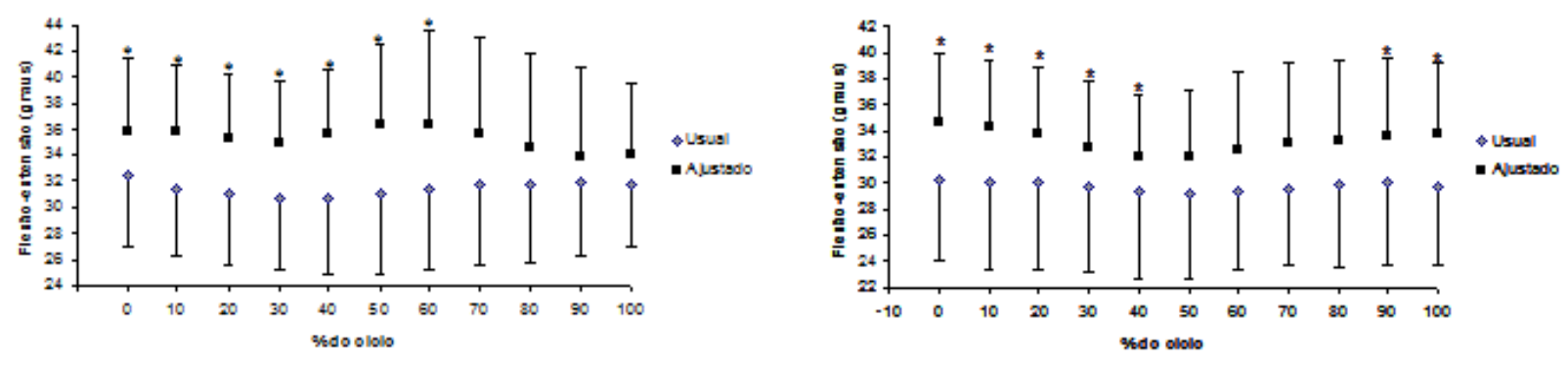

Segmento L3-L4
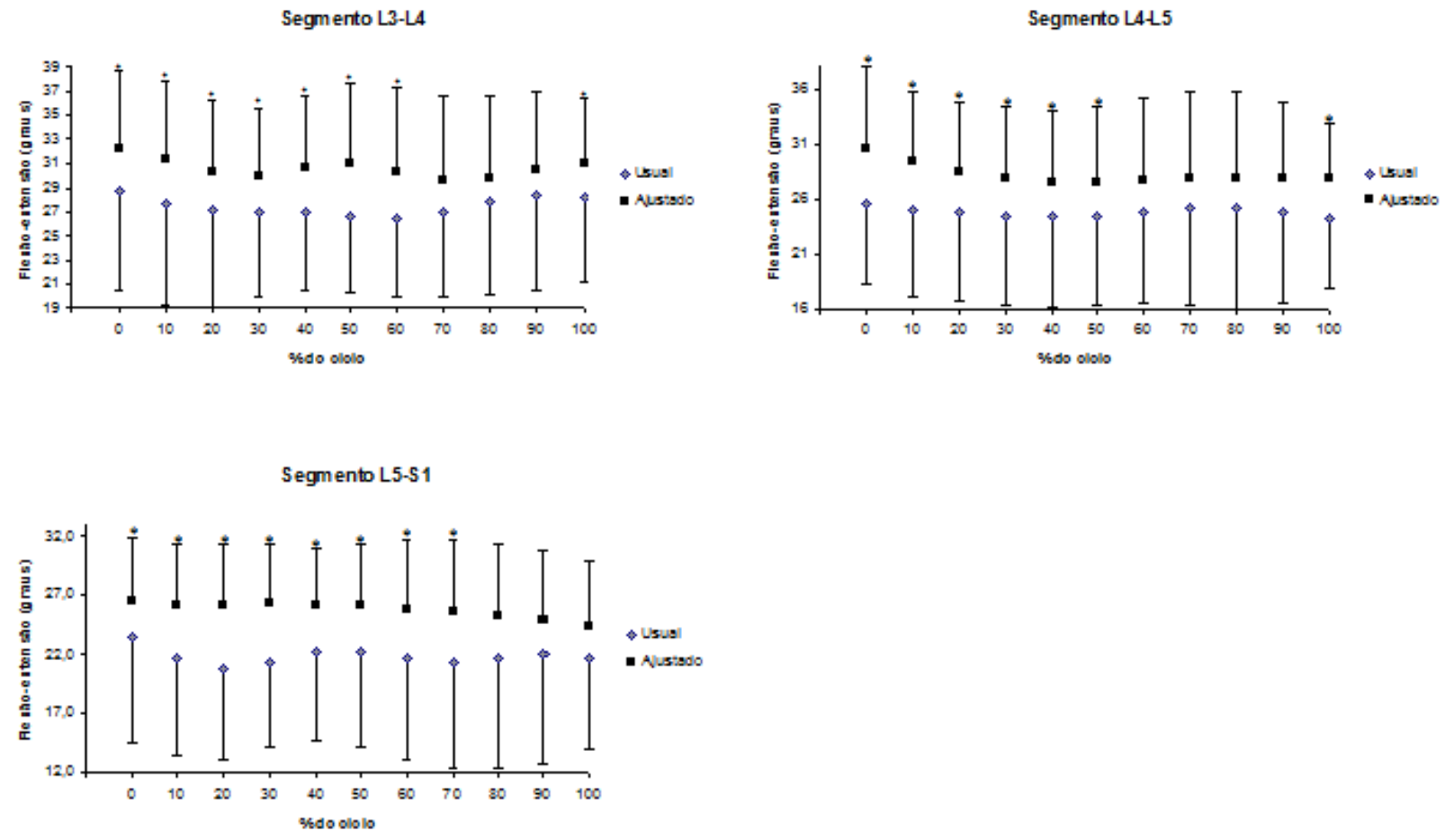

Figura 3. Comparação dos resultados de flexão-extensão da coluna lombar nas duas posições adotadas. * $\mathrm{p}<0,05$ representa valor estatisticamente significativo (Bonferronı) entre as situações de selim ajustado e usual.

Na Figura 3 são apresentadas as amplitudes de flexão/extensão para as regiões da coluna lombar analisadas. Percebeu-se maior amplitude de movimento na região inferior da lombar, principalmente na posição usual (posição ajustada: 2,5 para L1-L2, 2,6 $6^{\circ}$ para L2-L3, 2,7 para L3-L4, 2,9 $9^{\circ}$ para L4-L5 e 2,2 ${ }^{\circ}$ para L5-S1 e para a posição usual: $1,6^{\circ}$ para $L 1-L 2,1,0^{\circ}$ para L2-L3, 2,1 $1^{\circ}$ para L3-L4, 1,2 ${ }^{\circ}$ para L4-L5 e 2,7 para L5-S1), sendo que todos os segmentos apresentaram diferenças significativas na primeira metade do ciclo de pedalada (L1-L2 $0-60 \%$ (p1 $=0,006 ; \quad p 2=0,004 ; \quad p 3=0,005 ; \quad p 4=0,003$; p5=0,002; p6 $=0,005 ; p 7=0,023) ;$ L2-L3 $0-40 \%$ e $90-100 \% \quad(\mathrm{p} 1=0,009 ; \quad \mathrm{p} 2=0,004 ; \quad \mathrm{p} 3=0,002 ;$ $\mathrm{p} 4=0,003 ; \mathrm{p} 5=0,026 ; \mathrm{p} 10=0,041 ; \mathrm{p} 11=0,021)$; L3L4 $\quad 0-60 \%$ e $100 \% \quad(p 1=0,012 ; \quad p 2=0,015$; p3 $=0,022 ; \quad p 4=0,021 ; \quad p 5=0,011 ; \quad p 6=0,016$; p7 $=0,044 ; \quad p 11=0,034) ; \quad L 4-L 5 \quad 0-50 \%$ e $100 \%$ (p1 $=0,004 ; \quad \mathrm{p} 2=0,003 ; \quad \mathrm{p} 3=0,009 ; \quad \mathrm{p} 4=0,016$; $\mathrm{p} 5=0,012 ; \mathrm{p} 6=0,023 ; \mathrm{p} 11=0,016) ; \quad$ L5-S1 $0-70 \%$ $(p 1=0,004 ; p 2=0,004 ; p 3=0,004 ; p 4=0,004$; $\mathrm{p} 5=0,005 ; \mathrm{p} 6=0,015 ; \mathrm{p} 7=0,026 ; \mathrm{p} 8=0,045))$.

Os dados mostram que, para a inclinação lateral do segmento L1L2, houve efeito do ajuste $\left[F_{(1,7)}=0,938 ; \quad p=0,048\right] ; \quad$ e houve interações significativas entre ajuste e decil $\left[F_{(10,70)}=5,016\right.$; $p<0,001]$. Para a inclinação lateral do segmento L2L3, não houve efeito do ajuste $\left[F_{(1,7)}=0,007\right.$; $p=0,938]$; e não houve interações significativas entre ajuste e decil $\left[F_{(10,70)}=0,686 ; p=0,734\right]$. Para a inclinação lateral do segmento $L 3 L 4$, não houve efeito do ajuste $\left[F_{(1,7)}=0,002 ; p=0,970\right]$; e houve interações significativas entre ajuste e decil 
$\left[F_{(10,70)}=2,321 ; p=0,020\right]$. Para a inclinação lateral do segmento L4L5, houve efeito do ajuste $\left[F_{(1,7)}=0,912 ; \quad p=0,050\right] ; \quad$ e houve interações significativas entre ajuste e decil $\left[F_{(10,70)}=4,370\right.$; $p<0,001]$. Para a inclinação lateral do segmento L5S1, não houve efeito do ajuste $\left[F_{(1,7)}=2,626\right.$; $\mathrm{p}=0,149]$; e não houve interações significativas entre ajuste e decil $\left[F_{(10,70)}=0,640 ; p=0,775\right]$.

A Figura 4 ilustra em quais decis do ciclo do PDV foram encontradas diferenças significativas entre a posição usual do selim e posição de selim ajustado para a coluna lombar no plano frontal (inclinação lateral). Neste plano ocorreram diferenças significativas, apenas para o segmento L1-L2 a 30, 40, 80, 90 e $100 \%$ do ciclo ( $p 4=0,031$; p5=0,044; p9=0,014; p10=0,014; p11=0,044) e para o segmento L4-L5 a zero, 40 , e $100 \%$ do ciclo ( $\mathrm{p} 1=0,018 ; \mathrm{p} 5=0,039 ; \mathrm{p} 11=0,005)$, sendo encontrados valores médios superiores principalmente na posição usual nestes casos. Além disso, foi possível observar que em ambas as posições a maior inclinação lateral para a esquerda ocorreu na parte final do ciclo de pedalada, próximo ao PMS (100\% do ciclo) e a maior inclinação lateral para a direita ocorreu na metade do ciclo, próximo ao PMI, considerandose o PDV direito.

Na Figura 4 pode ser observada uma maior amplitude angular em relação à inclinação lateral (plano frontal) na região superior da lombar, principalmente na posição ajustada (posição ajustada: $19,0^{\circ}$ para L1-L2, 14,3 para L2-L3, $11,4^{\circ}$ para L3-L4, $11,0^{\circ}$ para L4-L5 e $12,1^{\circ}$ para L5-S1 e para a posição usual: $15,3^{\circ}$ para L1-L2, $14,4^{\circ}$ para L2-L3, 9,6 $6^{\circ}$ para L3-L4, 8,6 $6^{\circ}$ para L4-L5 e $10,7^{\circ}$ para L5-S1).
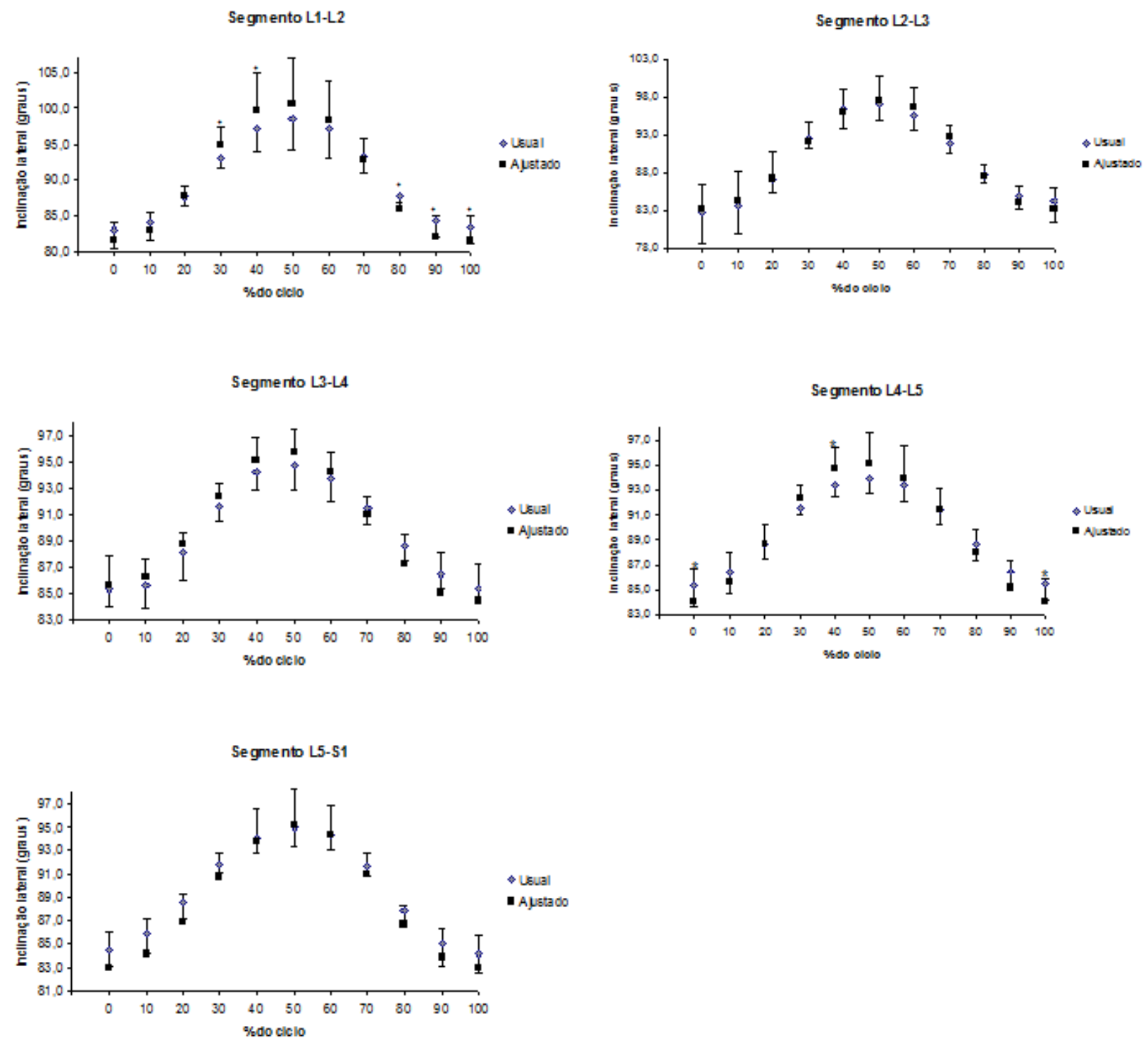

Figura 4. Comparação dos resultados de inclinação lateral da coluna lombar nas duas posições adotadas. * $p<0,05$ representa valor estatisticamente significativo (Bonferroni) entre as situações de selim ajustado e usual. 
Os dados mostram que, para a rotação da haste $L 1$, houve efeito do ajuste $\left[F_{(1,7)}=0,912\right.$; $p=0,050]$; e houve interações significativas entre ajuste e decil $\left[F_{(10,70)}=2,122 ; p=0,034\right]$. Para a rotação da haste $L 2$, não houve efeito do ajuste $\left[F_{(1,7)}=0,004 ; \quad p=0,950\right] ; \quad e$ houve interações significativas entre ajuste e decil $\left[\mathrm{F}_{(10,70)}=2,110\right.$; $\mathrm{P}=0,035]$. Para a rotação da haste $\mathrm{L3}$, houve efeito do ajuste $\left[F_{(1,7)}=0,912 ; p=0,050\right]$; e não houve interações significativas entre ajuste e decil $\left[F_{(10,70)}=0,797 ; p=0,632\right]$. Para a rotação da haste $L 4$, houve efeito do ajuste $\left[F_{(1,7)}=0,945 ; p=0,047\right]$; e não houve interações significativas entre ajuste e decil $\left[F_{(10,70)}=1,397 ; p=0,200\right]$. Para a rotação da haste $L 5$, houve efeito do ajuste $\left[F_{(1,7)}=2,006\right.$; $p=0,032]$; e houve interações significativas entre ajuste e decil $\left[F_{(10,70)}=2,571 ; p=0,010\right]$.
A Figura 5 ilustra em quais decis do ciclo do PDV foram encontradas diferenças significativas entre a posição usual do selim e posição de selim ajustado para a coluna lombar no plano transverso. Neste plano ocorreram diferenças significativas apenas para L1 em $20 \%$ do ciclo (p3=0,045), L3 a 90\% do ciclo (p10=0,05), L4 em zero e $10 \%$ do ciclo $(p 1=0,023 ; p 2=0,022)$ e para L5 em zero, 10, 20, 90, e 100\% do ciclo do PDV ( $\mathrm{p} 1=0,014 ; \quad \mathrm{p} 2=0,037 ; \quad \mathrm{p} 3=0,025 ; \quad \mathrm{p} 10=0,031$; $p 11=0,001)$, sendo encontrados valores médios superiores na posição usual. Nela pode-se observar que em ambas as posições a maior rotação para a esquerda ocorreu na parte final do ciclo de pedalada, próximo ao PMS, e a maior rotação para a direita ocorreu na metade do ciclo, próximo ao $\mathrm{PMI}$, considerando-se o PDV direito.
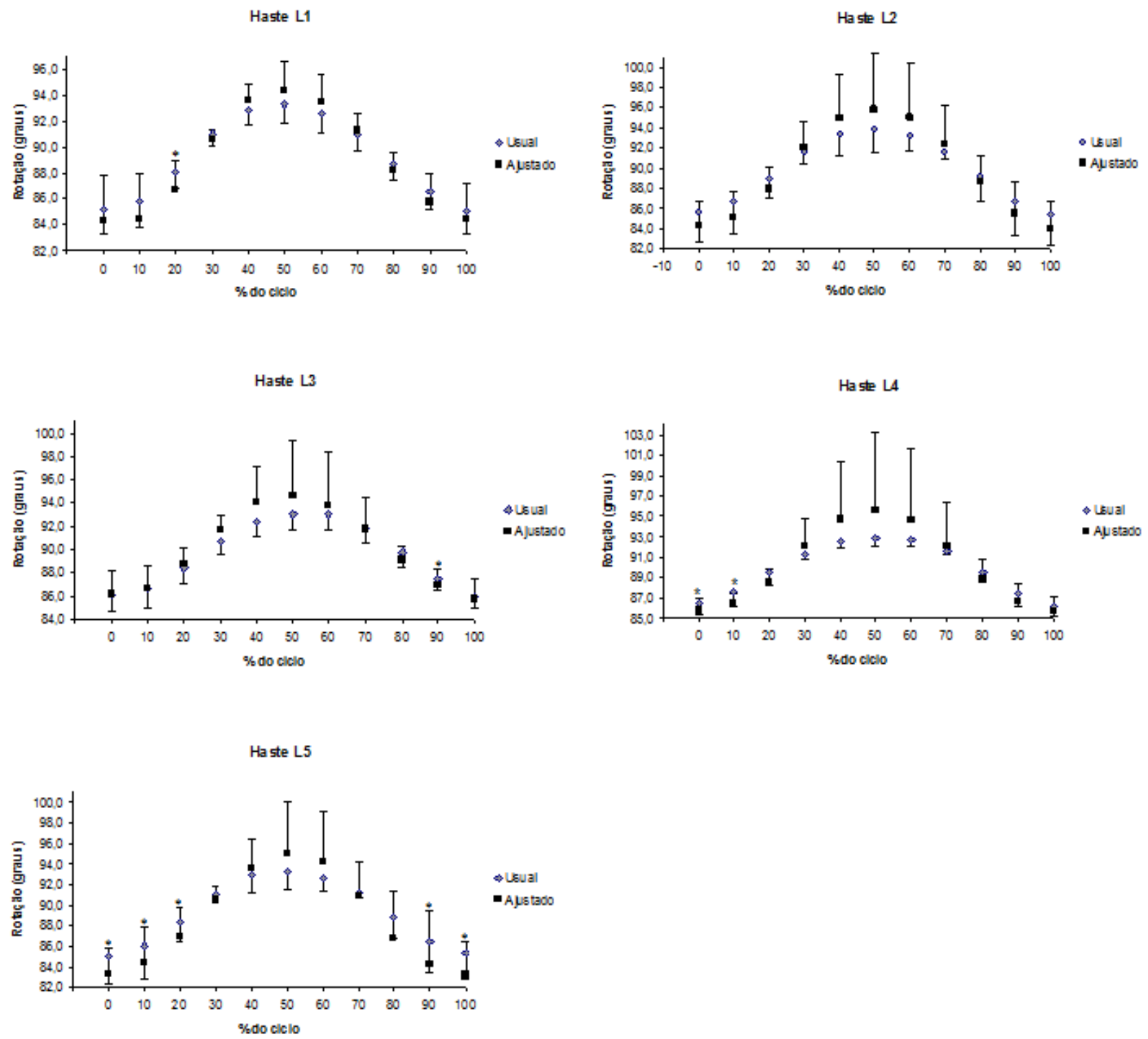

Figura 5. Comparação dos resultados de rotação da coluna lombar nas duas posições adotadas. * $\mathrm{p}<0,05$ representa valor estatisticamente significativo (Bonferronı) entre as situações de selim ajustado e usual. 
$\mathrm{Na}$ Figura 5 a amplitude de rotação apresentou-se maior em L2 e L5 em ambas as posições (posição ajustada: 10,1ำ para L1-L2, $11,9^{\circ}$ para L2-L3, 8,8 ${ }^{\circ}$ para L3-L4, 9,9 ${ }^{\circ}$ para L4-L5 e $11,8^{\circ}$ para L5-S1 e para a posição usual: $8,2^{\circ}$ para $L 1-L 2,8,5^{\circ}$ para $L 2-L 3,7,1^{\circ}$ para $L 3-L 4,6,7^{\circ}$ para L4-L5 e $8,3^{\circ}$ para L5-S1).

\section{Discussão}

\section{Comportamento angular da coluna lombar com os diferentes ajustes do selim}

$\mathrm{Na}$ literatura consultada, nenhum estudo teve o objetivo de comparar o comportamento angular da coluna com a utilização de diferentes ajustes da altura do selim, mas sim, com diferentes modelos de selim (BRESSEL; LARSON, 2003), diferentes angulações do selim (USABIAGAet al., 1997) e em diferentes posições de agarre no guidom (SAUERet al., 2007). No entanto, é no selim que os maiores erros no ajuste corporal do ciclista na bicicleta são encontrados (MARTINS et al., 2007).

Neste estudo, a elevação do selim aumentou a flexão da coluna lombar e acarretou maior anteversão pélvica (Figuras 2 e 3). Isso se deve ao fato da situação de selim ajustado aumentar a altura do selim, o que provoca maior flexão do tronco, de forma semelhante ao que ocorre quando se assume uma postura aerodinâmica na bicicleta. Isto vai ao encontro dos achados de Bressel e Larson (2003), onde a inclinação pélvica foi aumentada em $8 \%$ com a utilização do selim parcialmente vazado e em 16\% com a utilização do selim vazado em relação ao selim plano. Os ângulos de flexão do tronco foram maiores com a utilização do selim vazado em comparação com os outros modelos. As ciclistas apresentaram um aumento de $77 \%$ de anteversão pélvica e de $11 \%$ de flexão do tronco quando utilizaram a posição aerodinâmica em relação à posição mais elevada no guidom.

A amplitude de flexão encontrada no presente estudo, para ambas as posições, aumentou na direção de L4-L5 e diminuiu em L5-S1 na posição ajustada, sendo, no geral, maiores na posição ajustada (Figura 3). Este comportamento é semelhante ao encontrado por diversos estudos e, portanto, característicos do ciclismo, tanto recreacional quanto competitivo (BRESSEL; LARSON, 2003; SAUER et al., 2007; USABIAGA et al., 1997). Neste caso, a alteração na altura do selim não apresentou efeitos significantes sobre a cinemática da lombar.
Estudando as variações angulares da coluna lombar em diferentes posições adotadas em diferentes tipos de bicicletas, Usabiaga et al. (1997) encontraram que as posições envolveram uma mudança de lordose para cifose vertebral. A fim de obter uma posição mais aerodinâmica, os ciclistas flexionaram mais o tronco, alterando desta forma o ângulo do quadril, e posicionando a pelve na horizontal, sem alterar os ângulos intervertebrais. Um aumento gradativo das amplitudes angulares na posição aerodinâmica (de $3^{\circ} \mathrm{em} \mathrm{L1-L2} \mathrm{para} 6^{\circ} \mathrm{em} \mathrm{L4-L5),} \mathrm{decrescendo}$ em L5-S1 $\left(3^{\circ}\right)$ e na posição mais elevada de agarre do guidom, faz a amplitude manter-se semelhante entre os segmentos para 0 movimento de flexão-extensão $\left(4^{\circ}\right)$. Segundo os autores, as mudanças observadas podem modificar a biomecânica normal da coluna lombar, mas a carga mecânica global sobre a coluna é reduzida quando se apoia o peso sobre os membros superiores (posição aerodinâmica). Concluíram também que o ciclismo não gera forças biomecânicas prejudiciais à coluna lombar, até mesmo em condições de ciclismo profissional.

Os valores de inclinação lateral reportados no presente estudo variaram de 10 a $19^{\circ}$ na posição ajustada e de 8 a $15^{\circ}$ na posição usual (Figura 4), sendo que as maiores amplitudes ocorreram em L1-L2. Já os valores de rotação variaram de 8 a $11^{\circ}$ na posição ajustada e de 6 a $8^{\circ}$ na posição usual (Figura 5), sendo que as maiores amplitudes ocorreram em L2 e L5. Estas magnitudes angulares não agridem as estruturas que compõem a região lombar, pois estão dentro dos padrões esperados e também considerados normais, concordando com White e Panjabi (1978) e Usabiaga et al. (1997).

White e Panjabi (1978) apresentam as amplitudes dos segmentos da coluna nas três direções. A maior flexão-extensão em L1-L2 foi de $12^{\circ}$, aumentando para $19^{\circ} \mathrm{em}$ L5-S1, a inclinação lateral variou, mas diminuiu em direção a $\mathrm{S} 1$ e a rotação apresentou as menores amplitudes, sendo que em $S 1$ ocorreu a maior rotação $\left(4^{\circ}\right)$. Deve ser notado que o movimento no plano sagital origina-se primeiramente na região lombar, enquanto que a mobilidade neste plano geralmente diminui na extremidade inferior da coluna lombar comparada às regiões torácicas superiores (WHITE; PANJABI, 1978). No presente estudo, os valores de flexão também aumentaram na porção inferior, só que 
apresentaram valores menores (2,9॰ em L4-L5 na situação ajustada e de 2,7ํem L5-S1 na situação usual) (Figura 3), a inclinação lateral também diminuiu em direção a L5-S1 em ambas as situações, só que apresentaram valores maiores (19 na situação ajustada e 15ำ na situação usual em L1-L2) (Figura 4), enquanto que as maiores amplitudes de rotação ocorreram em L2 e L5 em ambas as situações (11ำ ajustado e $8^{\circ}$ usual) (Figura 5).

Os valores de amplitude de anteversão pélvica encontrados no presente estudo foram de $4,3^{\circ}$ para a situação ajustada e de $3,7^{\circ}$ para a situação usual (Figura 2), ou seja, semelhantes aos apresentados por Sauer et al. (2007), mas ambas um pouco superiores. Isto indica que a posição ajustada do selim permite maior amplitude de movimento pélvico. Sauer et al. (2007) encontraram excursões médias angulares da pelve variando em $2^{\circ}$ durante um ciclo de pedalada - na inclinação pélvica anterior, na posição de descanso - a $8^{\circ}$ na rotação internaexterna na posição aerodinâmica. Variações angulares maiores foram observadas com a pelve rodando internamente e lateralmente para a posição aerodinâmica. Os dados do presente estudo demonstraram que maiores valores de flexão ocorreram na situação ajustada enquanto que as maiores médias de inclinação e rotação ocorreram na situação de ajuste usual, evidenciando a importância da utilização do ajuste correto da altura do selim, pois naquela não se exige tanto da coluna lombar quanto nesta.

\section{Caracterização e quantificação do comportamento angular da coluna lombar durante o ciclo da pedalada}

Durante o andar, a forma geométrica da coluna adapta-se e oscila, realizando um ciclo completo por passada no plano frontal (inclinação lateral) e dois no sagital (flexão-extensão) (CALLAGHAN; PATLA; MCGILL, 1999; SAUNDERS et al., 2005). Este comportamento foi semelhante durante uma revolução do ciclo do pedal no presente estudo tanto para a coluna lombar quanto para a pelve (Figuras 2 a 5). Estes resultados corroboram com o estudo de White e Panjabi (1978), demonstrando as possibilidades de movimentação da coluna vertebral em relação aos seus graus de liberdade, tanto em valores absolutos, quanto considerando características de comportamento angular.

No ciclismo, a rotação pélvica é importante porque influencia a aerodinâmica, a eficiência e o risco de lesões por excesso de treino (SAUER et al., 2007). Em nosso estudo, esta variável se mostrou sensível a alteração da posição do selim. Os resultados sugeriram que o ajuste do selim aumenta a amplitude angular desta variável $\left(8,3^{\circ}\right.$ na situação usual e 11,8ำ na situação ajustada, Figura 5). Para Mueller, McEvoy e Everett (2005), a amplitude angular alcançada durante longo período sentado no ciclismo pode ter pequena relevância para a posição que o ciclista utiliza nesta modalidade, encontrando valores superiores aos do presente estudo. $\underline{\text { Campos }}$ (2005) sugere que esta diferença se deva ao fato dos ciclistas recreacionais pedalarem em cadência moderada (70 rpm), já que o movimento da pelve e da coluna são provocados pelo movimento dos membros inferiores.

Embora avaliações cinemáticas na corrida tenham proposto metodologias para análise dos movimentos de rotação da coluna, especialmente da região lombar (BRENZIKOFER et al., 2000; DE PAULA et al., 2009), poucos estudos descrevem estratégias para tal monitoramento no ciclismo. Em nosso estudo aplicamos um protocolo de medição com base na fixação de hastes sobre as vértebras lombares e os resultados revelaram que durante a pedalada, um comportamento parecido com o encontrado para a marcha (SCHACHE et al., 2002; WHITTLE; LEVINE, 1999) é observado para a coluna lombar. No presente estudo, o comportamento de flexão lombar (Figura 3) foi semelhante ao de anteversão pélvica (Figura 2), sendo que na situação de selim ajustado (Figura 3) esta coordenação foi mais consistente, ou seja, a flexão lombar seguiu o movimento pélvico estreitamente ao longo do ciclo. Whittle e Levine (1999) encontraram que, no plano sagital, a mudança na lordose lombar durante o ciclo da marcha foi consistente para cada sujeito, mas variou consideravelmente entre eles, também durante a coordenação de fase entre a inclinação pélvica e a lordose lombar. No plano frontal, houve um padrão muito mais consistente de movimento, a inclinação lombar lateral geralmente seguiu o padrão de obliquidade pélvico, apenas 
antes do contato inicial, sobreposta na relação geralmente linear. O plano transversal mostrou oscilação semelhante entre rotação axial da pelve e rotação axial da coluna lombar, a não ser pelo movimento pélvico ser um pouco maior, acontecendo depois no ciclo da marcha que o movimento da coluna lombar. A lordose lombar teve seu próprio padrão de movimento, não sendo simplesmente um reflexo do movimento pélvico, seguindo o movimento pélvico de perto no plano frontal.

O estudo do movimento pélvico durante 0 ciclismo utilizando análise de vídeo foi objetivo de estudo de Neptune e Hull (1995). No eixo X (inclinação lateral) as maiores amplitudes foram encontradas entre 0 segundo e 0 terceiro quadrantes do ciclo $\left(180^{\circ}\right)$, e as menores amplitudes no início e no final do ciclo. Para o eixo Z (rotação) o movimento característico foi semelhante ao encontrado por Sauer et al. (2007), sendo maior para a direita no início e no final do ciclo e menor na metade do mesmo. Este comportamento foi semelhante ao encontrado no presente estudo, para a anteversão pélvica (Figura 2) e para a inclinação lateral (Figura 4) e rotação lombar (Figura 5) em ambas as situações avaliadas, indicando que este comportamento é característico do ciclismo.

Sauer et al. (2007) encontraram variações angulares maiores com a pelve rodando internamente $\left(\sim 3^{\circ}\right)$ e lateralmente $\left(\sim 2^{\circ}\right)$ para a posição aerodinâmica, atingindo ângulos de inclinação lateral e rotação interna máxima perto do ponto morto inferior (PMI) do ciclo de pedalada. A inclinação anterior da pelve oscilou duas vezes em um ciclo de pedalada com a máxima inclinação anterior correspondente à posição do ponto morto superior (PMS) do PDV. A posição aerodinâmica causou no quadril inclinação na direção anterior, mas não alterou substancialmente a forma das excursões angulares, o que concorda com Saunders et al. (2005) e com o presente estudo. Sauer et al. (2007) encontraram ainda, para homens na posição de descanso, rotação pélvica média de $5^{\circ}$, inclinação lateral média de $4^{\circ}$ e anteversão pélvica média de $12^{\circ}$ e na posição aerodinâmica anteversão pélvica média de $20^{\circ}$. Quanto ao comportamento pélvico ao longo do ciclo de pedalada, ocorreu maior flexão no início, meio e fim do ciclo, enquanto que a maior inclinação lateral e rotação ocorreram ambos no meio do ciclo $\left(180^{\circ}\right.$ ou PMI). Resultados muito semelhantes a estes foram encontrados por Schache et al. (2002), ao longo de um ciclo de passada, avaliando o comportamento angular da coluna lombar durante a corrida e aos encontrados no presente estudo avaliando o ciclo de pedalada. Isso sugere grande similaridade no comportamento angular da região lombar comparando a marcha e o ciclismo.

Burnett et al. (2004), comparando a cinemática da coluna em ciclistas com e sem dor lombar crônica inespecífica, mostram que a cinemática da coluna não diferiu entre os mesmos ao longo do ciclo de pedalada. Estes dados apresentaram amplitudes menores às encontradas no estudo de Usabiaga et al. (1997) e também no presente estudo. A utilização de uma bicicleta desajustada pode provocar a ocorrência de desconfortos e dores corporais, dentre elas, dor lombar, pélvica e cervical que podem ser agravadas devido à prática continuada e, devido à ocorrência de desconforto ou dor, ocorrem ações antecipatórias que limitam a amplitude de movimento (CALLAGHAN; PATLA; MCGILL, 1999). Dessa forma, menores amplitudes de movimento poderiam ter relações com o uso da bicicleta desajustada, o que aumentaria o risco de lesão.

White e Panjabi (1978) sugerem que, embora a coluna tóraco-lombar possa ser considerada uma única estrutura, o movimento no plano sagital origina-se primeiramente na região lombar, enquanto que a mobilidade neste plano geralmente diminui na extremidade inferior da coluna lombar comparada às regiões torácicas superiores. No presente estudo, os valores de inclinação lateral também diminuíram em direção a L5-S1 em ambas as situações, só que apresentaram valores maiores (Figura 4), a flexão aumentou na porção inferior, só que apresentou valores menores (Figura 3 ), enquanto que as maiores amplitudes de rotação ocorreram em L2 e L5 em ambas as situações (Figura 5).

Van Herp et al. (2000) encontraram valores de flexão bem superiores aos encontrados no presente estudo, enquanto que os valores de inclinação lateral e de rotação foram semelhantes, indicando que o ciclismo não provoca movimentos biomecanicamente desfavoráveis que sobrecarreguem a coluna lombar e que agridam as estruturas que compõem esta região, pois os mesmos estão dentro dos padrões esperados e também considerados normais, mesmo com 0 
selim desajustado, concordando também com White e Panjabi (1978) e Usabiaga et al. (1997).

No presente estudo (Figuras 2 e 3), uma maior variabilidade foi observada, em média, nos resultados obtidos para a flexão/extensão. Isto pode estar diretamente relacionado com 0 número e a distribuição dos pontos de referência utilizados (BRENZIKOFER et al., 2000), e também com o tipo de movimento analisado, que ocorre no plano sagital e perfaz dois ciclos completos por passada (CALLAGHAN; PATLA; MCGILL, 1999; SAUNDERS et al., 2005) e também durante um ciclo de pedalada, como pode-se observar no estudo de Sauer et al. (2007). Já para movimentos de inclinação lateral (Figura 4) notam-se maiores amplitudes nesta região da coluna, indo de encontro as observações de Brenzikofer et al. (2000). Poucos estudos tratam dos movimentos de rotação axial nesta região, sendo que os valores encontrados (Figura 5) vão ao encontro dos apresentados por White e Panjabi (1978), Neptune e Hull (1995), Usabiaga et al. (1997) e Sauer et al. (2007). Quanto ao comportamento ao longo do ciclo encontrado no presente estudo, pode-se perceber que ocorreram dois ciclos de flexão-extensão durante um ciclo completo do PDV e apenas um ciclo de inclinação lateral e de rotação neste mesmo período, fato que demonstra a existência de grandes semelhanças entre o comportamento angular lombar durante a marcha e o ciclismo. Dentre as limitações do presente estudo podemos citar o tamanho da amostra e o fato de apenas uma carga de trabalho ter sido testada.

\section{Conclusão}

Quanto ao comportamento ao longo do ciclo, foi encontrado que a flexão teve um comportamento diferenciado, ou seja, ocorreram dois ciclos de flexão-extensão durante um ciclo completo do PDV enquanto ocorreu apenas um ciclo de inclinação lateral ou de rotação neste mesmo período, demonstrando maior regularidade neste tipo de movimento.

Em relação às médias angulares, a flexão foi maior na situação ajustada enquanto que as maiores médias de inclinação e rotação ocorreram na situação usual de ajuste do selim, evidenciando a importância da utilização do ajuste correto do mesmo. Os valores de rotação aqui encontrados, tanto na situação usual quanto ajustada, não ultrapassaram os valores fisiológicos, indicando que nenhuma dessas situações de ajuste possa prejudicar a coluna lombar.

\section{Referências}

BRENZIKOFER, R.; BARROS, R. M. L.; LIMA FILHO, E. C.; TOMA, E.; BORDINI, L. S. Alterações no dorso e coluna vertebral durante a marcha. Revista Brasileira de Biomecânica, São Paulo, v. 1, n. 1, p. 21-26, 2000. Disponível em:

$<$ http://citrus.uspnet.usp.br/biomecan/ojs/index.ph p/rbb/issue/view/1>. Acesso em: 21 ago. 2006.

BRESSEL, E.; LARSON, B. J. Bicycle seat designs and their effect on pelvic angle, trunk angle and comfort. Medicine and Science in Sports and Exercise, Indianápolis, v. 35, n. 2, p. 327-332, 2003. doi:

http://dx.doi.org/?10.1249/01.MSS.0000048830.22 $\underline{964.7 \mathrm{c}}$

BURKE, E. R. High-Tech Cycling: the faster rider. Colorado Springs: Human Kinetics, 1996.

BURKE, E. R.; PRUITT, A. L. Body positioning for cycling. In: BURKE, E. R. (ed) High-Tech Cycling. 2 ed. p. 69-92. Champaign: Ed. Human Kinetics, 2003.

BURNETT, A. F.; CORNELIUS, M. W.; DANKAERTS, W.; O'SULLIVAN P. B. Spinal kinematics and trunk muscle activity in cyclists: a comparison between healthy controls and nonspecific chronic low back pain subjects - a pilot investigation. Manual Therapy, Londres, v. 9, p. 211-219, 2004. doi:

http://dx.doi.org/10.1016/j.math.2004.06.002

CALLAGHAN, J. P.; PATLA, A. E.; MCGILL, S. M. Low back three-dimensional joint forces, kinematics, and kinetics during walking. Clinical Biomechanics, Huddersfield, v. 14, p. 203-216, 1999. doi: http://dx.doi.org/10.1016/S0268$\underline{0033(98) 00069-2}$

CAMPOS, M. H. Adaptações geométricas da coluna vertebral durante a marcha. Dissertação de Mestrado. Universidade Estadual de Campinas - Faculdade de Educação Física. 2005.

DE PAULA, M. C.; CAMPOS, M. H.; MEIRELLES, A. A.; MORAES, M. A. A.; BRENZIKOFER, R. Análise da amplitude de movimentação da coluna vertebral, frequência e comprimento da passada durante a marcha e a corrida. Revista Brasileira de Biomecânica, São Paulo, v. 10, n. 18, p. 1-8, 2009. Disponível em:

$<$ http://citrus.uspnet.usp.br/biomecan/ojs/index.ph p/rbb/issue/view/22>. Acesso em: 12 dez. 2009. 
DOREL, S.; COUTURIER, A.; HUG, F. Influence of different racing positions on mechanical and electromyographic patterns during pedaling.Scandinavian Journal of Medicine \& Science in Sports, Copenhagen, p 1-11, 2007.doi: http://dx.doi.org/10.1111/i.16000838.2007.00765.x

GREGOR, R. J.; RUGG, S. G. Effects of saddle height and pedaling cadence on power output and efficiency. In: Science of Cycling, BURKE, E. R. (ed.). CHAMPAIGNS, IL: Human Kinetics Publishers, p. 69-90, 1986.

MARTINS, E. A.; DAGNESE, F.; KLEINPAUL, J. F.; CARPES, F. P.; MOTA, C. B. Avaliação do posicionamento corporal no ciclismo competitivo e recreacional. Revista Brasileira de Cineantropometria\& Desempenho Humano, Florianópolis, v. 9, p.183-188, 2007. Disponível em:

$<$ http://www.rbcdh.ufsc.br/MostraEdicao.do?edica o=30>. Acesso em: 26 fev. 2008.

MUELLER, P.; MCEVOY, M.; EVERETT, S.The long sitting screening test in elite cyclists. Journal of Science and Medicine in Sport, Queensland, v. 8, n. 4, p. 369-374, 2005.Disponível em: $<$ http://www.jsams.org/article/S14402440\%2805\%2980051-9/pdf >. Acesso em: 02 dez. 2006.

NEPTUNE, R. R.; HULL, M. L. Accuracy assessment of methods for determining hip movement in seated cycling.Journal of

Biomechanics, Oxford, v. 28, n. 4, p. 423-437, 1995.Disponívelem:

$<$ http://www.jbiomech.com/article/00219290\%2894\%2900080-N/pdf>. Acesso em: 02 dez. 2006.

ROWE, T.; HULL, M. L.; WANG, E. L. A pedal dynamometer for off-road bicycling. Journal of Biomechanics Engineering, Pittsburg, v. 120, n. 1, p. 160-164, 1998.Disponívelem: $<$ http://scitation.aip.org/Biomechanical/>.Acessoe m: 15 set. 2000 .

SAUER, J. L.; POTTER, J. J.; WEISSHAAR, C. L.; PLOEG, H. L.; THELEN D. G. Influence of gender, power, and hand position on pelvic motion during seated cycling. Medicine \& Science in Sports \& Exercise, Baltimore, v. 39, n. 12, p. 2204-2211, 2007.doi: http://dx.doi.org/10.1249/mss.0b013e3181568b66

SAUNDERS, S. W.; SCHACHE, A.; RATH, D.; HODGES, P. W. Changes in three dimensional lumbo-pelvic kinematics and trunk muscle activity with speed and mode of locomotion. Clinical Biomechanics, Huddersfield, v. 20, p. 784-93, 2005. doi: http://dx.doi.org/10.1016/i.clinbiomech.2005.04.00 $\underline{4}$

SCHACHE, A. G.; BLANCH, P.; RATH, D.; WRIGLEY, T.; BENNELL, K. Three-dimensional angular kinematics of the lumbar spine and pelvis during running. Human Movement Science, Amsterdam, v. 21, p. 273-293, 2002. doi:http://dx.doi.org/10.1016/S01679457(02)00080-5

USABIAGA, J.; CRESPO, R.; IZA, I.; ARAMENDI, J.; TERRADOS, N.; POZA, J. J. Adaptation of the Lumbar Spine to Different Positions in Bicycle Racing. Spine, Philadelphia, v. 22, n. 17, p. 19651969, 1997.Disponível em:

$<$ http://journals.Iww.com/spinejournal/Abstract/199 7/09010/Adaptation of the Lumbar Spine to Dif ferent.4.aspx>. Acesso em: 23 set. 2000.

VAN HERP, G.; ROWE, P.; SALTER, P.; PAUL, J. $P$. Three-dimensional lumbar spinal kinematics: a study of range of movement in 100 healthy subjects aged 20 to $60+$ years. Rheumatology, Toronto, v. 39, p. 1337-1340, 2000. doi: http://dx.doi.org/10.1093/rheumatology/39.12.1337

WHITE, A. A.; PANJABI, M. M. Clinical biomechanics of the spine. Philadelphia, J. B. Lippincott, v. 667, p. 110, 1978.

WHITTLE, M. W.; LEVINE, D. Tree-dimensional relationships between the movements of the pelvis and lumbar spine during normal gait. Human Movement Science, Amsterdam, v. 18, p. 681692, 1999. doi:http://dx.doi.org/10.1016/S0167$\underline{9457(99) 00032-9}$

Agradecimentos: apoio financeiro CAPES.

\section{Endereço:}

Julio Francisco Kleinpaul

Rua 286, 453 Meia Praia

Itapema SC Brasil

88220-000

Telefone: (47) 9171-7520

e-mail: juliofk@gmail.com

Recebido em: 20 de outubro de 2010.

Aceito em: 10 de dezembro de 2012.

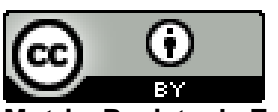

Motriz. Revista de Educação Física. UNESP, Rio Claro, SP, Brasil - elSSN: 1980-6574 - está licenciada sob Creative Commons - Atribuição 3.0 